\title{
Baker's Cyst with Intramuscular Extension into Vastus Medialis Muscle
}

\author{
Tae Wan Kim, $\mathrm{MD}^{1}$, Jeung Tak Suh, $\mathrm{MD}^{1}$, Seung Min Son, $\mathrm{MD}^{1}$, Tae Yong Moon, $\mathrm{MD}^{2}$, In Sook Lee, $\mathrm{MD}^{2}$,
} Kyung Un Choi, $\mathrm{MD}^{3}$ and Jeung Il Kim, $\mathrm{MD}^{1}$

Departments of ${ }^{1}$ Orthopaedic Surgery, ${ }^{2}$ Radiology, ${ }^{3}$ Pathology, Pusan National University School of Medicine, Busan, Korea

Baker's cysts are one of the most common cystic lesions around the knee joint and mainly caused by fluid distension of the gastrocnemiussemimembranous bursa that is situated along the medial side of the popliteal fossa. Typically, a Baker's cyst extends along the intermuscular planes around the knee joint and may enlarge any direction. However, it is mostly located in the inferomedial or superficial layers of the knee joint and less commonly extends laterally or proximally. Expansion of the cyst tends to respect the intermuscular planes, and Baker's cysts along the intramuscular route have been rarely reported. Thus, we report a case of Baker's cyst with intramuscular extension into the vastus medialis muscle.

Key words: Baker's cyst, Knee joint, Intramuscular extension, Vastus medialis muscle.

Baker's cysts are one of the most common cystic lesions around the knee joint that can be produced by an escape of fluid from the knee joint into the gastrocnemius-semimembranous bursa through normal communication or herniation of the synovial membrane through the posterior knee capsule ${ }^{1,2)}$. Magnetic resonance imaging (MRI) evidence of Baker's cysts is seen in $4.7 \%$ to $19 \%$ of the patients with symptoms of internal knee derangement ${ }^{3,4)}$.

In general, a Baker's cyst results from egress of joint fluid through the weakened posteromedial capsule into the gastrocnemius-semimembranous bursa. It is commonly located in the inferomedial or superficial layers of the knee joint and rarely extends laterally or proximally ${ }^{5,6}$. Bursae have a tendency

Received March 27, 2012; Revised (1st) April 26, 2012;

(2nd) July 6, 2012; Accepted July 16, 2012.

Correspondence to: Jeung Il Kim, MD.

Department of Orthopaedic Surgery, Pusan National University School of Medicine, 179 Gudeok-ro, Seo-gu, Busan 602-739, Korea.

Tel: +82-51-240-7248, Fax: +82-51-247-8395

Email: osteokim@yahoo.co.kr

This is an Open Access article distributed under the terms of the Creative Commons Attribution Non-Commercial License (http://creativecommons.org/licenses/by-nc/3.0/) which permits unrestricted non-commercial use, distribution, and reproduction in any medium, provided the original work is properly cited. to enlarge along the intermuscular planes, and thus intramuscular Baker's cysts have not yet been reported domestically and have rarely been seen in the international literature ${ }^{7)}$.

We report a rare case of Baker's cyst with intramuscular extension into the vastus medialis muscle that was confirmed by MRI and removed by excision biopsy with a review of the literature.

\section{Case Report}

A 37-year-old female patient visited our institution complaining of a painless mass in the posteromedial aspect of the left knee that had been present for several months. The physical examination showed mild knee swelling and a $5-\mathrm{cm}$ diameter mass along the medial side of the popliteal fossa. There was no tenderness or feeling of pressure around the mass, neurological symptoms, and range of motion (ROM) limitation. The patient had neither history of illness nor trauma. Plain radiography revealed no abnormal findings, such as calcification. A lobulated cyst measuring $5 \times 5 \times 3 \mathrm{~cm}$ was identified in the vastus medialis muscle by MRI. The cyst showed a low signal intensity on T1weighted images whereas an intermediate-high signal intensity on T2-weighted images. The inhomogeneity in signal intensity was attributed to hemorrhage within the cyst (Fig. 1). There was no opening or stalk for direct communication between the cyst and joint cavity or combined pathology, such as meniscus tears, on MRI. Therefore, an open excisional biopsy was performed. 

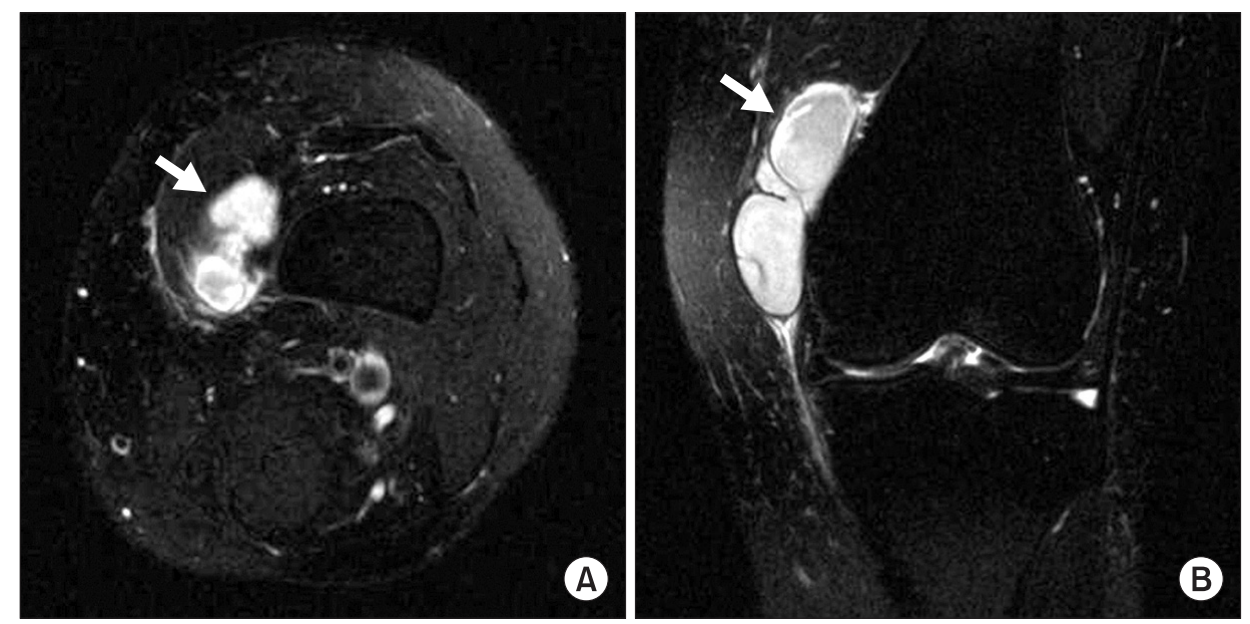

Fig. 1. A Baker's cyst with intramuscular extension into vastus medialis muscle. Axial T2-weighted image showing a high-signal or intermediate-signal intensity of the cyst (arrow) enveloped by the vastus medialis muscle (A). Coronal T2-weighted image showing the multilocular intramuscular extension through the vastus medialis muscle (arrow) (B).
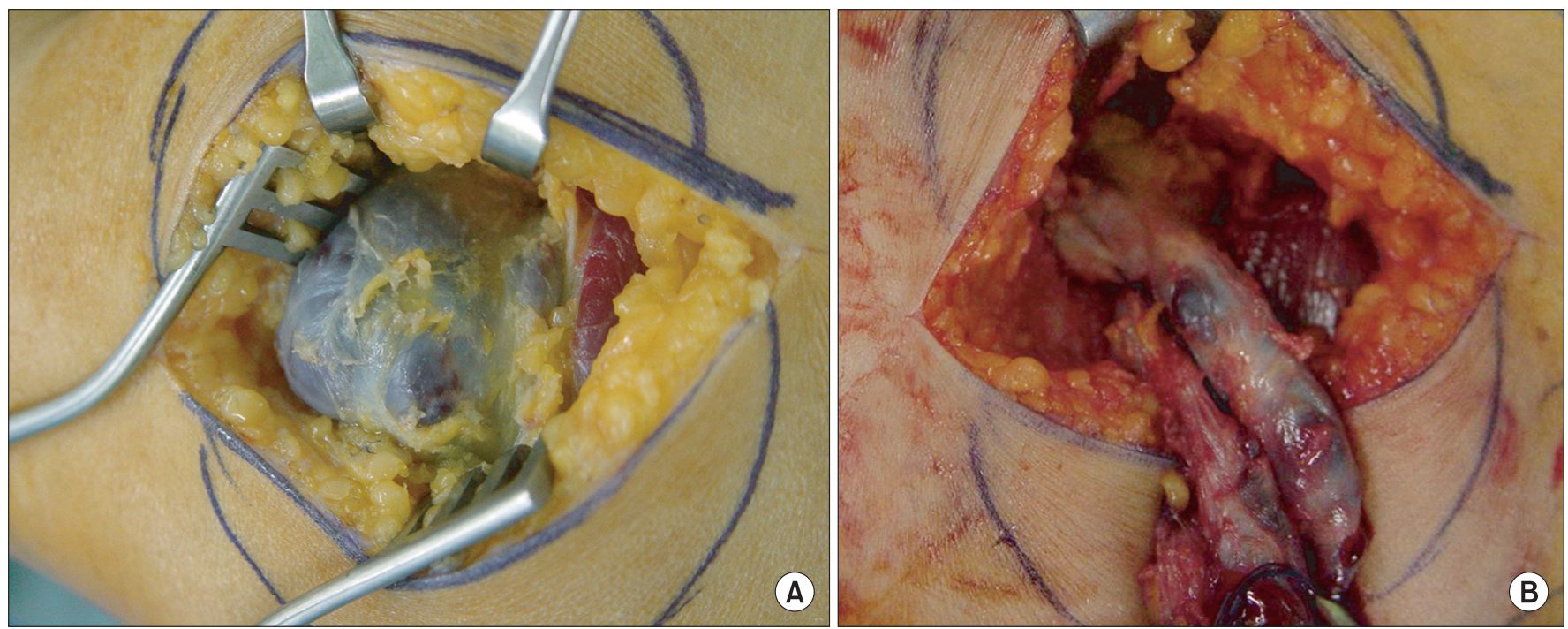

Fig. 2. Intraoperative photograph showing a Baker's cyst with bloody contents in the vastus medialis muscle (A). The cyst has an intraarticular communication with knee joint (B).

With the patient under spinal anesthesia, a longitudinal skin incision was made along the medial side of the left knee and soft tissue release was done to expose the cyst. The location of the cyst in the vastus medialis muscle was confirmed after incising the fascia. The cyst was filled with dark brown fluid suggestive of hemorrhage within the cyst (Fig. 2A). During detachment from the surrounding tissues, communication between the cyst and the joint cavity was noted (Fig. 2B). After complete removal of the cyst (Fig. 3), an opening for intraarticular communication with the joint cavity was observed and $\geq 5 \mathrm{~mm}$ fenestration of the opening in the posterior joint capsule was done to block unidirectional passage of fluid (Fig. 4). Histopathological examination showed that the cyst wall was surrounded by synovial membrane and composed of thick fibrous tissue, which led us to diagnose a Baker's cyst.

\section{Discussion}

Baker's cysts result from fluid distension of the gastrocnemiussemimembranous bursa ${ }^{1,2)}$. With the increasing use of MRI in the diagnosis of cysts, significant correlations between the development of cysts and the presence of intraarticular lesions have been reported in many studies ${ }^{8)}$. Thus, intraarticular lesions are considered as an important factor in cyst formation. In a prospective study by Rupp et al. ${ }^{9)}$, the prevalence of intraarticular lesions was significantly higher in patients with popliteal cysts. Of the 100 patients who were scheduled for arthroscopy, popliteal cysts were observed in $20 \%$, of which $85 \%$ had chondral lesions 


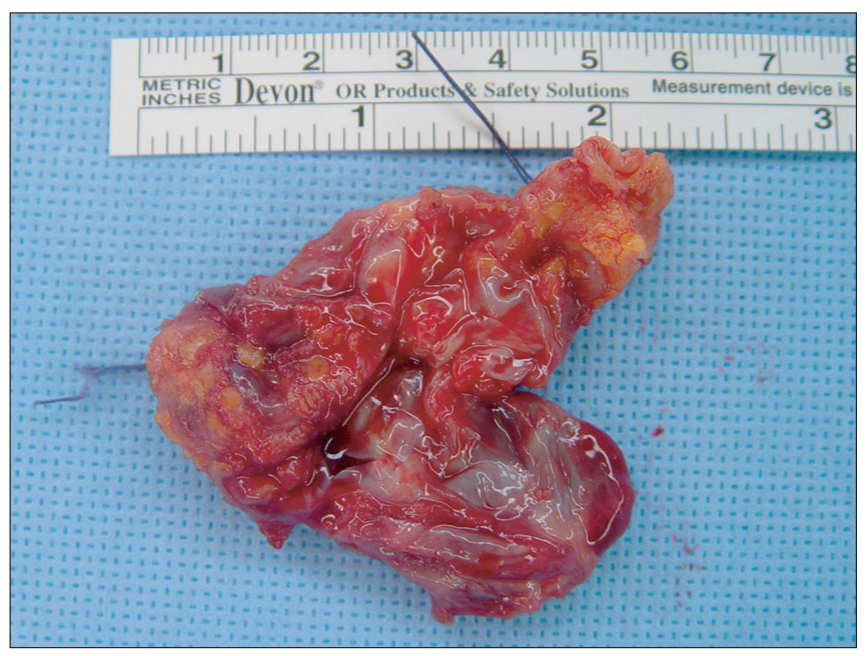

Fig. 3. The excised Baker's cyst is shown.

and $70 \%$ had medial meniscal tears. In particular, lesions in the posterior part of the medial meniscus are most commonly present in patients with Baker's cysts ${ }^{10}$. In the presence of a posterior horn medial meniscus tear, the septum between the gastrocnemius-semimembranous bursa that abuts the posterior horn becomes thinner and more fragile, which results in the formation of a connection between the joint cavity and the bursa. Anatomical studies have demonstrated that the fluid that is injected through this channel is trapped in the bursa, resulting in the formation of a popliteal cyst ${ }^{8}$.

Baker's cysts are mostly asymptomatic and often found incidentally during MRI examinations for internal derangement of the knee. Common clinical presentations include painless swelling in the medial popliteal fossa that changes in size during knee movements, local pain, a feeling of pressure in the popliteal fossa, ROM limitation, and symptoms related to adjacent tissue compression.

Available diagnostic modalities for Baker's cysts and associated intraarticular lesions include ultrasonography, arthroscopy, computed tomography, and MRI. Ultrasonography is useful for determining the location, size, and contents of a Baker's cyst, identifying the presence of a septum and multiplicity, and assessing relationships to adjacent structures. In particular, it is reliable for differentiation of Baker's cysts from popliteal artery aneurysm, thrombophlebitis that presents with swelling, and solid mass in the popliteal fossa. MRI is the currently preferred non-invasive diagnostic tool that provides high quality images for accurate assessment of the affected intraarticular structures and lesions and cyst location ${ }^{8}$.

The incidence of the presence of an opening between the

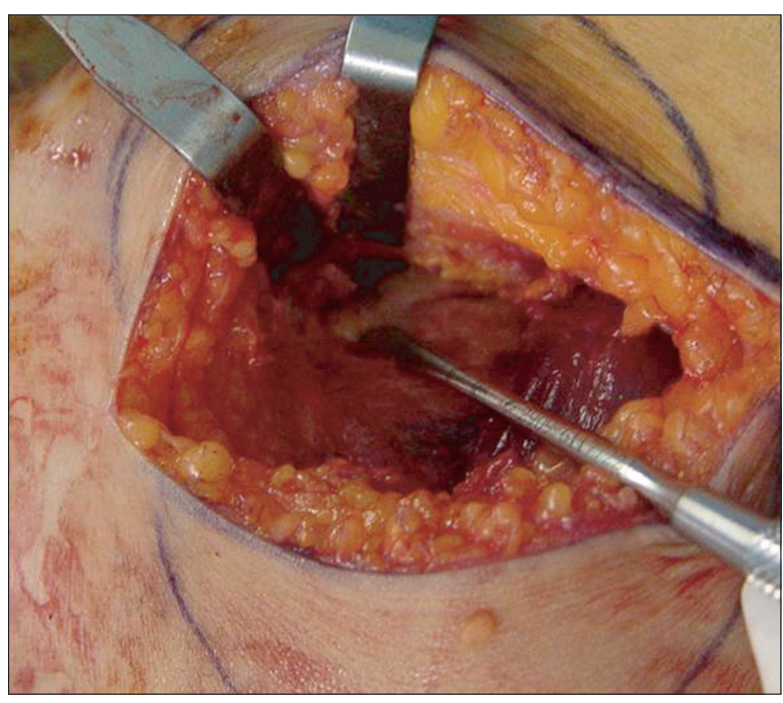

Fig. 4. After complete excision of the cyst, an opening for intraarticular communication with the knee joint was seen, and fenestration of the opening was done.

Baker's cyst and joint cavity varies from $57 \%$ to $99 \%$. Johnson et al. ${ }^{11)}$ reported it as $37 \%$ and age, trauma, degenerative arthritis, rheumatoid arthritis, effusion, and meniscal lesions were influencing factors ${ }^{12)}$. Johnson et al. ${ }^{11)}$ reported that an opening to the cyst was observed in the transverse band in Type 3 posterior medial wall that was classified according to the anatomic configuration. In our case, the presence of an opening or stalk for direct communication between the cyst and joint cavity was not revealed on preoperative MRI scan but was identified during dissection from the adjacent tissues for open excisional biopsy. Lindgren ${ }^{13)}$ advocated that a valvular mechanism in the channel that is responsible for the one-way passage of fluid from the joint cavity to a cyst should be removed during surgery to prevent persistence and recurrence of cysts. According to Sansone and De Ponti ${ }^{14)}$, the goal of surgical treatment for popliteal cysts is to remove intraarticular lesions and block the one-way passage of fluid to the cyst. To close the channel between the knee cavity and the cyst, Childress ${ }^{10)}$ used gastrocnemius and semimembranosus tendons, Rauschning ${ }^{15)}$ chose the tendon of the medial head of the gastrocnemius as a pedicle graft, and Hughston et al. ${ }^{12)}$ performed a simple capsulorrhaphy. However, Sansone and De Pont ${ }^{14)}$ reported that these techniques may impede accurate capsular suturing because it is difficult to expose the capsular gap due to the superimposition of the gastrocnemius and semimembranosus. Lindgren ${ }^{13)}$ reported that the articular capsule may not be able to resist the intraarticular pressure during flexion-extension movements of the knee. In addition, Lindgren and Willen ${ }^{16)}$ showed that a communication exists 
between the knee joint and the gastrocnemius-semimembranous bursa in $50 \%$ of normal patients without a popliteal cyst. Sansone and De Ponti ${ }^{14)}$ observed neither complications nor weakened articular structures after enlargement of the capsular orifice. In our case, we performed $\geq 5 \mathrm{~mm}$ fenestration of the opening in the posterior capsule to eliminate the unidirectional flow of the fluid. It is our understanding that re-establishment of bidirectional communication would prevent collection of fluid in the bursa and even if there is an accumulation of fluid, it will be absorbed automatically due to the open excision of the cyst.

A Baker's cyst may enlarge in any direction. It is generally located in the inferomedial or superficial layers of the knee joint and rarely extends laterally or proximally ${ }^{5,6)}$. Bursae have a tendency to enlarge along the intermuscular planes, and thus intramuscular Baker's cysts have not yet been reported domestically and have rarely been seen in the international literature. In 2004, Fang et al. ${ }^{7)}$ reported a case of Baker's cyst in the vastus medialis muscle that has communication with the knee joint cavity via a narrow stalk between the gastrocnemius and the semimembranosus and two cases of the cyst within the gastrocnemius medialis muscle.

The Baker's cyst in the vastus medialis muscle we presented in this report indicates that the cyst can enlarge intramuscularly. In general, cysts are localized to intermuscular areas because they grow along the weak areas of the body. In our patient, the cyst extended intramuscularly through the weakened fascia. Damage to the fascia is caused by direct or indirect trauma typically in the superficial layer of muscle. However, it is theoretically possible to occur in the deep layer of a muscle. Our patient had no history of direct or indirect trauma and we could not identify any damage or perforation in the fascia of the vastus medialis muscle during surgery, but the cyst was filled with dark brown fluid suggesting hemorrhage. On the other hand, a cyst may enlarge intramuscularly due to increased intraarticular pressure in the presence of a congenital fascial defect that originates in the area where vessels and nerves penetrate the fascia ${ }^{17)}$.

Baker's cysts typically enlarge along the intermuscular planes. In this report, we presented a case of Baker's cyst with intramuscular extension into the vastus medialis muscle that was confirmed on MRI scan. The cyst was treated successfully by open excisional biopsy in the absence of MRI evidence of a stalk in the cyst.

\section{Acknowledgements}

This study was supported by a grant of the Korean Heath Technology R\&D Project, Ministry of Health and Welfare,
Republic of Korea (A070001).

\section{References}

1. Bickel WH, Burleson RJ, Dahlin DC. Popliteal cyst; a clinicopathological survey. J Bone Joint Surg Am. 1956;38: 1265-74.

2. Rauschning W. Anatomy and function of the communication between knee joint and popliteal bursae. Ann Rheum Dis. 1980;39:354-8.

3. Miller TT, Staron RB, Koenigsberg T, Levin TL, Feldman F. MR imaging of Baker cysts: association with internal derangement, effusion, and degenerative arthropathy. Radiology. 1996;201:247-50.

4. Fielding JR, Franklin PD, Kustan J. Popliteal cysts: a reassessment using magnetic resonance imaging. Skeletal Radiol. 1991;20:433-5.

5. Steiner E, Steinbach LS, Schnarkowski P, Tirman PF, Genant HK. Ganglia and cysts around joints. Radiol Clin North Am. 1996;34:395-425.

6. Torreggiani WC, Al-Ismail K, Munk PL, Roche C, Keogh C, Nicolaou S, Marchinkow LP. The imaging spectrum of Baker's (Popliteal) cysts. Clin Radiol. 2002;57:681-91.

7. Fang CS, McCarthy CL, McNally EG. Intramuscular dissection of Baker's cysts: report on three cases. Skeletal Radiol. 2004;33:367-71.

8. Sansone V, de Ponti A, Paluello GM, del Maschio A. Popliteal cysts and associated disorders of the knee. Critical review with MR imaging. Int Orthop. 1995;19:275-9.

9. Rupp S, Seil R, Jochum P, Kohn D. Popliteal cysts in adults. Prevalence, associated intraarticular lesions, and results after arthroscopic treatment. Am J Sports Med. 2002;30:112-5.

10. Childress HM. Popliteal cysts associated with undiagnosed posterior lesions of the medial meniscus. The significance of age in diagnosis and treatment. J Bone Joint Surg Am. 1970;52:1487-92.

11. Johnson LL, van Dyk GE, Johnson CA, Bays BM, Gully SM. The popliteal bursa (Baker's cyst): an arthroscopic perspective and the epidemiology. Arthroscopy. 1997;13:6672.

12. Hughston JC, Baker CL, Mello W. Popliteal cyst: a surgical approach. Orthopedics. 1991;14:147-50.

13. Lindgren PG. Gastrocnemio-semimembranosus bursa and its relation to the knee joint. III. Pressure measurements in joint and bursa. Acta Radiol Diagn (Stockh). 1978;19:37788. 
14. Sansone V, De Ponti A. Arthroscopic treatment of popliteal cyst and associated intra-articular knee disorders in adults. Arthroscopy. 1999;15:368-72.

15. Rauschning W. Popliteal cysts (Baker's cysts) in adults. II. Capsuloplasty with and without a pedicle graft. Acta Orthop Scand. 1980;51:547-55.
16. Lindgren PG, Willen R. Gastrocnemio-semimembranosus bursa and its relation to the knee joint. I. Anatomy and histology. Acta Radiol Diagn (Stockh). 1977;18:497-512.

17. Braunstein JT, Crues JV 3rd. Magnetic resonance imaging of hereditary hernias of the peroneus longus muscle. Skeletal Radiol. 1995;24:601-4. 